\title{
Solução direta para um problema inverso de autovalores e autovetores de segunda ordem
}

\author{
João Batista Carvalho \\ Depto de Matemática Pura e Aplicada, UFRGS \\ Porto Alegre, RS \\ E-mail: carvalho @mat.ufrgs.br
}

\begin{abstract}
Resumo: Este trabalho apresenta uma estratégia de solução, que testes computacionais têm mostrado dar bons resultados, para o problema inverso de autovalores de sistemas de vibração lineares de segunda ordem, onde é desejado atualizar as matrizes de massa, amortecimento e rigidez a partir de medições de parte das frequências naturais e respectivos modos de vibração. A estratégia emprega resultados sobre ortogonalidade envolvendo o espectro de sistemas de vibração lineares de segunda ordem; além disso, o ajuste de modelo proposto é um método direto que possibilita um excelente desempenho computacional por ser rico em operações com blocos de matrizes. Exemplo numérico é apresentado.
\end{abstract}

Palavras-chave: ajuste de modelo, autovalores, segunda ordem

\section{Introdução}

Sistemas de vibração governados por equações diferenciais lineares de segunda ordem podem ser descritos, usando variáveis de estado, pelo sistema de equações diferenciais

$$
M \ddot{q}(t)+C \dot{q}(t)+K q(t)=f(t),
$$

onde $M, C, K \in \mathbb{R}^{n \times n}$ são chamadas, respectivamente, de matrizes de inércia, de amortecimento e de rigidez. As matrizes $M$ e $K$ são simétricas, $M$ é normalmente positiva definida, enquanto $K$ é normalmente simétrica semi-definida. $C$ é uma matriz simétrica. Quantidades $q$ e $\dot{q}$ são as variáveis de estado do sistema. A quantidade $f(t)$ representa a ação de forças externas sobre o sistema no tempo $t$.

Matematicamente, a existência de soluções vibratórias, isto é, da forma $q_{i}(t)=x_{i} e^{\lambda_{i} t}$, $x_{i} \in \mathbb{R}^{n}$ não-nulo, requer que $\left(\lambda_{i}, x_{i}\right), x_{i} \neq 0$, seja solução de

$$
\lambda^{2} M x+\lambda C x+K x=0
$$

onde $\lambda=\lambda_{i}$ é chamado de autovalor e $x=x_{i}$ é um autovetor associado a $\lambda_{i}$; essas quantidades estão associadas as frequências naturais e aos respectivos modos de vibração do sistema em (1).

Muito frequentemente, a representação $(M, C, K)$ de um sistema de vibrações é obtida através do método de elementos finitos. Nesse contexto, normalmente algumas simplificações de modelagem precisam ser feitas, tanto nas equações de cada elemento da discretização, quanto nas suas condições de contorno. Como consequência, pode haver divergências entre propriedades previstas pela representação analítica construída, que aqui chamaremos de $\left(M_{a}, C_{a}, K_{a}\right)$ e propriedades reais do sistema.

Uma estratégia que foi primeiramente empregada no caso de sistemas de vibração nãoamortecidos $\left(C_{a}=C_{u}=0\right)$, cujas equações governantes são redutíveis as de primeira ordem

$$
\sigma M x=K x,
$$


onde $\sigma$ é escalar, consiste em ajustar a representação $\left(M_{a}, K_{a}\right)$ para $\left(M_{u}, K_{u}\right)$ usando informação obtida pela medição de um grupo de $p$ frequências naturais do sistema, e respectivos modos de vibração, na esperança que propriedades associadas aos valores medidos sejam incorporadas a nova representação. Essa estratégia também vem sendo aplicada no caso amortecido. Muito frequentemente, entretanto, o processo descrito acima ocasiona mudanças indesejáveis nas frequências e modos de vibrações que não foram diretamente envolvidos na medição, isto é, no resto do espectro do sistema. A mudança no espectro não-medido, como consequência do ajuste de modelo, é conhecida como spill-over. Em muitas situações, a ocorrência de spill-over não representa restrição ao uso da representação $\left(M_{u}, C_{u}, K_{u}\right)$; em outras, por exemplo, há divergência sobre a própria característica de estabilidade do sistema, uma vez que autovalores que representam frequências naturais não medidas acabam sendo movidos para o lado direito do plano complexo.

Nas últimas três décadas, o problema de ajuste de modelo tem recebido muita atenção, e muitas estratégias têm sido propostas para tratá-lo. Uma revisão bastante abrangente pode ser encontrada em [1]. Por outro lado, uma revisão bastante introdutória pode ser encontrada em [2]. No início da década de 1980, multiplicadores de Lagrange foram introduzidos por Baruch [3] e Bermann-Nagy [4]. Esses métodos, de maneira geral e no contexto de sistemas não-amortecidos, supõem que alguma das matrizes de inércia ou de rigidez está correta, enquanto a outra deve ser corrigida. Métodos tipicamente matriciais foram introduzidos por Caesar [5] e Linck et al. [6], onde basicamente foi tratado o problema de aproximar as inversas das matrizes de inércia e rigidez (atualizadas) a partir dos dados experimentais. Técnicas oriundas de problemas de controle, como realocação de estruturas (eigenstructure assignment) foram propostas por Zimmerman e Widengren [7] e Inman e Minas [8]. Esses últimos métodos determinam controles que são capazes de mudar as frequências e modos da representação analítica de maneira a ajustá-la aos dados medidos.

Esses primeiros métodos, apesar de serem diretos e computacionalmente muito eficientes, não conseguem preservar relevantes propriedades do sistema original, o que muitas vezes prejudica a própria validade da aplicação do novo modelo analítico obtido. Com o intuito de apresentar uma metodologia capaz de preservar a estrutura da matriz de rigidez do sistema, Kabe [9], Caesar e Peter [10], e outros, desenvolveram algoritmos capazes de preservar a conetividade daquela matriz, mas que podiam produzir indesejado spill-over sobre o espectro não medido.

Em [11], resultados que possibilitaram uma abordagem mais robusta para o problema do spill-over foram apresentados. Como consequência, Carvalho et al [12] propôs um método direto para ajuste de modelo em sistemas não-amortecidos, que garantidamente não produz spillover, mas que não consegue preservar as conetividades da matriz de rigidez, e tampouco sua positividade. Chu et al. $[13,14,15]$ consideraram modelos amortecidos em conexão com as propriedades de não produzir spill-over e manter simetria e positividade das matrizes $M$ e $K$; seus resultados tornaram mais claro quais hipóteses deviam acompanhar a formulação dos problemas de atualização de modelo, amortecidos ou não, para garantir a existência de soluções com relevância prática. Mao e Dai [16] também desenvolveram método para ajuste capaz de preservar positividade e garantidamente não produzir spill-over.

Este trabalho usa resultados de [11] para desenvolver, no contexto de sistemas amortecidos, fórmulas parametrizadas para $M_{u}, C_{u}$ e $K_{u}$ que garantidamente não produzem spill-over, enquanto o problema de incorporar os dados medidos, equivalente a solução de um sistema sobredeterminado de equações algébricas, é resolvido via técnica de mínimos quadrados.

Considere a decomposição do espectro analítico conhecido

$$
M X_{1} \Lambda_{1}^{2}+C X_{1} \Lambda_{1}+K X_{1}=0
$$

onde $X_{1} \in \mathbb{R}^{n \times p}$ é a matriz formada a partir dos $p$ modos de vibração analíticos, $\Lambda_{1} \in \mathbb{R}^{p \times p}$ é matriz quasidiagonal cujos autovalores são as quantidades $\lambda_{1}, \lambda_{2}, \ldots, \lambda_{p}$ associadas as frequências naturais analíticas. Além disso, são também conhecidas matrizes $Y_{1} \in \mathbb{R}^{n \times p}$, formada a partir dos $p$ modos de vibração medidos, e $\Sigma_{1} \in \mathbb{R}^{p \times p}$ que é quasidiagonal e possui como autovalores as quantidades $\mu_{i}$ associadas as $p$ frequências naturais medidas. Conhecida técnica de 
descomplexificação, necessária para tratar o caso de autovalores complexos para que as matrizes envolvidas sejam todas reais, é descrita em [15]. O problema de ajuste de modelo procura encontrar matrizes $M_{u}, C_{u}, K_{u}$ tais que

$$
M_{u} Y_{1} \Sigma_{1}^{2}+C_{u} Y_{1} \Sigma_{1}+K Y_{1}=0
$$

e dessa forma incorporar as medições em $Y_{1}, \Sigma_{1}$ ao novo modelo, substituindo a informação em $X_{1}$ e $\Lambda_{1}$. Além disso, o espectro da nova representação $\left(M_{u}, C_{u}, K_{u}\right)$ deve possuir os mesmos pares $\left(\lambda_{i}, x_{i}\right)$ que não foram atualizados a partir de $\left(M_{a}, C_{a}, K_{a}\right)$, sem que sua informação seja necessária, entretanto. Na seção 2, apresentamos resultados que permitem tratar adequadamente o problema de impedir a ocorrência de spill-over, usando parametrização adequada. Na seção 3 , tratamos da questão de estabelecer solução generalizada para o problema de incorporar os dados medidos. Na seção 4, empregamos a estratégia proposta em um exemplo numérico.

\section{Ortogonalidade do espectro e fórmulas que impedem spill-over}

Considere a decomposição espectral da representação analítica $\left(M_{a}, C_{a}, K_{a}\right)$ :

$$
\begin{aligned}
& M_{a} X_{1} \Lambda_{1}^{2}+C_{a} X_{1} \Lambda_{1}+K_{a} X_{1}=0 \\
& M_{a} X_{2} \Lambda_{2}^{2}+C_{a} X_{2} \Lambda_{2}+K_{a} X_{2}=0
\end{aligned}
$$

onde $X_{1}$ e $\Lambda_{1}$, definidos na seção anterior, são conhecidos, e $X_{2}$ e $\Lambda_{2}$, que representam o espectro não-medido, não são necessariamente conhecidos. Considere também matrizes $Y_{1}, \Sigma_{1}$, também definidas na seção anterior. Seja $\Omega(U)$ o conjunto de autovalores de uma matriz $U$.

Lema 2.1. Seguindo [11], supomos que os autovalores $\lambda_{1}, \ldots, \lambda_{2 n}$ são distintos e não-nulos. Supomos também que existe uma decomposição espectral

$$
M X \Lambda^{2}+C X \Lambda+K X=0
$$

onde $X \in \mathbb{R}^{n \times 2 n}, \Lambda \in \mathbb{R}^{2 n \times 2 n}$, para um modelo $(M, C, K)$. Sob essas condições, é demonstrado em [11] que existem matrizes diagonais em blocos $D_{1}, D_{2}, D_{3}$ tais que

$$
\begin{aligned}
(X \Lambda)^{T} M X \Lambda-X^{T} K X & =D_{1} \\
(X \Lambda)^{T} C X \Lambda+(X \Lambda)^{T} K X+X^{T} K X \Lambda & =D_{2} \\
(X \Lambda)^{T} M X+X^{T} M X \Lambda+X^{T} C X & =D_{3}
\end{aligned}
$$

Corolário 2.1. Seja $\Omega\left(\Lambda_{1}\right) \bigcap \Omega\left(\Lambda_{2}\right)=\emptyset$ e uma decomposição espectral de segunda ordem

$$
M\left[\begin{array}{ll}
X_{1} & X_{2}
\end{array}\right]\left[\begin{array}{ll}
\Lambda_{1}^{2} & \\
& \Lambda_{2}^{2}
\end{array}\right]+C\left[\begin{array}{ll}
X_{1} & X_{2}
\end{array}\right]\left[\begin{array}{ll}
\Lambda_{1} & \\
& \Lambda_{2}
\end{array}\right]+K\left[\begin{array}{ll}
X_{1} & X_{2}
\end{array}\right]=0 .
$$

Então as relações (9)-(11) acima implicam nas relações de ortogonalidade

$$
\begin{array}{r}
\left(X_{2} \Lambda_{2}\right)^{T} M X_{1} \Lambda_{1}-X_{2}^{T} K X_{1}=0 \\
\left(X_{2} \Lambda_{2}\right)^{T} C X_{1} \Lambda_{1}+\left(X_{2} \Lambda_{2}\right)^{T} K X_{1}+X_{2}^{T} K X_{1} \Lambda_{1}=0 \\
\left(X_{2} \Lambda_{2}\right)^{T} M X_{1}+X_{2}^{T} M X_{1} \Lambda_{1}+X_{2}^{T} C X_{1}=0 .
\end{array}
$$

Teorema 2.1. Dados $\Lambda_{1} \in \mathbb{R}^{p \times p}$ e $X_{1} \in \mathbb{R}^{n \times p}$ tais que $\Omega\left(\Lambda_{1}\right) \bigcap \Omega\left(\Lambda_{2}\right)=\emptyset$ e

$$
M X_{1} \Lambda_{1}^{2}+C X_{1} \Lambda_{1}+K X_{1}=0
$$

então para qualquer matriz simétrica $\Phi \in \mathbb{R}^{p \times p}$

$$
\begin{array}{r}
\triangle M=-M X_{1} \Lambda_{1} \Phi \Lambda_{1}^{T} X_{1}^{T} M \\
\triangle C=M X_{1} \Lambda_{1} \Phi X_{1}^{T} K+K X_{1} \Phi \Lambda_{1}^{T} X_{1}^{T} M \\
\triangle K=-K X_{1} \Phi X_{1}^{T} K
\end{array}
$$


garantidamente temos

$$
(M+\triangle M) X_{2} \Lambda_{2}^{2}+(C+\triangle C) X_{1} \Lambda_{2}+(K+\triangle K) X_{2}=0
$$

ou seja, o respectivo ajuste para $(M+\triangle M, C+\triangle C, K+\triangle K)$ mantém o espectro não medido.

\section{Demonstração:}

Seja $\left(X_{2}, \Lambda_{2}\right)$ formado pelo espectro não incluído em $\left(X_{1}, \Lambda_{1}\right)$. Então

$$
\begin{gathered}
(M+\triangle M) X_{2} \Lambda_{2}^{2}+(C+\triangle C) X_{2} \Lambda_{2}+(K+\triangle K) X_{2}=\left(M-M X_{1} \Lambda_{1} \Phi \Lambda_{1}^{T} X_{1}^{T} M\right) X_{2} \Lambda_{2}^{2}+(C+ \\
\left.M X_{1} \Lambda_{1} \Phi X_{1}^{T} K+K X_{1} \Phi \Lambda_{1}^{T} X_{1}^{T} M\right) X_{2} \Lambda_{2}+\left(K-K X_{1} \Phi X_{1}^{T} K\right) X_{2}= \\
-M X_{1} \Lambda_{1} \Phi \Lambda_{1}^{T} X_{1}^{T} M X_{2} \Lambda_{2}^{2}+M X_{1} \Lambda_{1} \Phi X_{1}^{T} K X_{2} \Lambda_{2}+K X_{1} \Phi \Lambda_{1}^{T} X_{1}^{T} M X_{2} \Lambda_{2}-K X_{1} \Phi X_{1}^{T} K X_{2}= \\
M X_{1} \Lambda_{1} \Phi\left(-\Lambda_{1}^{T} X_{1}^{T} M X_{2} \Lambda_{2}+X_{1}^{T} K X_{2}\right) \Lambda_{2}-K X_{1} \Phi\left(\Lambda_{1}^{T} X_{1}^{T} M X_{2} \Lambda_{2}-X_{1}^{T} K X_{2}\right)=0
\end{gathered}
$$

pela primeira relação de ortogonalidade (13) aplicada aos últimos termos entre parênteses.

Teorema 2.2. No contexto do Teorema 2.1, uma matriz $\Phi^{*} \in \mathbb{R}^{p \times p}$ define um modelo atualizado $\left(M_{u}, C_{u}, K_{u}\right)$ que incorpora os valores medidos $Y_{1}, \Sigma_{1}$ se e somente se $\Phi^{*}$ é solução simétrica do sistema linear sobredeterminado

$$
A_{1} \Phi B_{1}+A_{2} \Phi B_{2}=F
$$

onde

$$
\begin{array}{r}
A_{1}=M X_{1} \Lambda_{1}, B_{1}=\left(\Lambda_{1}^{T} X_{1}^{T} M Y_{1} \Sigma_{1}-X_{1}^{T} K Y_{1}\right) \Sigma_{1} \\
A_{2}=K X_{1}, B_{2}=X_{1}^{T} K Y_{1}-\Lambda_{1}^{T} X_{1}^{T} M Y_{1} \Sigma_{1} \\
F=M Y_{1} \Sigma_{1}^{2}+C Y_{1} \Sigma_{1}+K Y_{1} .
\end{array}
$$

\section{Demonstração:}

$$
\begin{gathered}
M_{u} Y_{1} \Sigma_{1}^{2}+C_{u} Y_{1} \Sigma_{1}+K Y_{1}=0 \Leftrightarrow\left(M-M X_{1} \Lambda_{1} \Phi \Lambda_{1}^{T} X_{1}^{T} M\right) Y_{1} \Sigma_{1}^{2}+\left(C+M X_{1} \Lambda_{1} \Phi X_{1}^{T} K+\right. \\
\left.K X_{1} \Phi \Lambda_{1}^{T} X_{1}^{T} M\right) Y_{1} \Sigma_{1}+\left(K-K X_{1} \Phi X_{1}^{T} K\right) Y_{1}=0
\end{gathered}
$$

e após distribuirmos os produtos e fatorarmos $M X_{1} \Lambda_{1}$ e $K X_{1}$

$$
\begin{gathered}
M X_{1} \Lambda_{1} \Phi\left[\Lambda_{1}^{T} X_{1}^{T} M Y_{1} \Sigma_{1}-X_{1}^{T} K Y_{1}\right] \Sigma_{1}+K X_{1} \Phi\left[X_{1}^{T} K Y_{1}-\Lambda_{1}^{T} X_{1}^{T} M Y_{1} \Sigma_{1}\right]= \\
M Y_{1} \Sigma_{1}^{2}+C Y_{1} \Sigma_{1}+K Y_{1}
\end{gathered}
$$

e o resultado segue.

\section{Solução generalizada para o problema de ajuste de modelo}

Conforme resultado da seção anterior, o ajuste de modelo via equações (17) - (19) não produz spill-over. Dentro da liberdade que existe para definir uma matriz $\Phi \in \mathbb{R}^{p \times p}$ simétrica, devemos fazê-lo de maneira a resolver (21) no sentido generalizado, pois claramente existem mais equações do que incógnitas em (21).

Nesta seção, $e_{i} \in \mathbb{R}^{p}$ denota a $i$-ésima coluna da matriz identidade em $\mathbb{R}^{p \times p} ; \operatorname{vec}(U)$ denota a forma vetor coluna de uma matriz $U ; \Omega((M, C, K))$ denota o conjunto de autovalores do modelo descrito pela tripla de matrizes $(M, C, K) ;\|U\|_{F}$ denota a norma Frobenius de uma matriz $U$; $\delta_{i j}$ denota o delta de Kronecker.

Observamos que matrizes simétricas $L_{i j} \in \mathbb{R}^{p \times p}$ definidas por

$$
L_{i j}=\left\{\begin{array}{ll}
e_{i} e_{i}^{T} & , j=i \\
\frac{e_{i} e_{j}^{T}+e_{j} e_{i}^{T}}{\sqrt{2}} & , j<i
\end{array}, i=1,2, \ldots, j ; j=1,2, \ldots, p\right.
$$


satisfazem a relação

$$
\operatorname{vec}\left(L_{i j}\right)^{T} \operatorname{vec}\left(L_{u v}\right)=\delta_{i u} \delta_{j v} .
$$

Portanto, o correspondente conjunto de vetores $\left\{\operatorname{vec}\left(L_{i j}\right)\right\}$ acima constitui uma base ortonormal de $\mathbb{R}^{P}$, onde $P=p(p+1) / 2$. Da mesma forma, as matrizes definidas em (25) formam uma base para o espaço das matrizes simétricas em $\mathbb{R}^{p \times p}$, e toda e qualquer matriz simétrica $\Phi \in \mathbb{R}^{p \times p}$ pode ser escrita como uma combinação linear

$$
\Phi=\alpha_{11} L_{11}+\alpha_{12} L_{12}+\alpha_{22} L_{22}+\cdots+\alpha_{p 1} L_{p 1}+\cdots+\alpha_{p p} L_{p p}
$$

de $P$ matrizes simétricas em $\mathbb{R}^{p \times p}$. Aplicando (27) em (21), obtemos que (21) equivale a

$$
\left[\begin{array}{lllllll}
\operatorname{vec}\left(R_{11}\right) & \operatorname{vec}\left(R_{12}\right) & \operatorname{vec}\left(R_{22}\right) & \operatorname{vec}\left(R_{13}\right) & \operatorname{vec}\left(R_{23}\right) & \ldots & \operatorname{vec}\left(R_{p p}\right)
\end{array}\right]\left[\begin{array}{c}
\alpha_{11} \\
\alpha_{12} \\
\alpha_{22} \\
\ldots \\
\alpha_{p p}
\end{array}\right]=\operatorname{vec}(F)
$$

que é um sistema com $n p$ equações e $P$ incógnitas, onde

$$
R_{i j}=A_{1} L_{i j} B_{1}+A_{2} L_{i j} B_{2} \in \mathbb{R}^{n \times p} ; \quad i=1,2, \ldots, j ; j=1,2, \ldots, p .
$$

A melhor solução do sistema de equações (28), no sentido dos mínimos quadrados, pode ser encontrada usando técnicas clássicas de matemática numérica, que normalmente empregam decomposição em valores singulares (S.V.D.) ou fatoração QR com pivotamento de colunas. Em qualquer desses dois casos, superior desempenho computacional pode ser atingido usando bibliotecas especializadas como LAPACK $[17,18]$, uma vez que a computação é muito rica em operações com blocos de matrizes [18]. Finalmente, $\Phi^{*}$ é encontrada avaliando (27), e então $M_{u}$, $C_{u}$ e $K_{u}$ são encontradas usando (17)-(19).

\section{Algoritmo 3.1:}

Entrada: matrizes $M_{a}, C_{a}, K_{a}, X_{1}, \Lambda_{1}, Y_{1}, \Sigma_{1}$

Saída: matrizes $M_{u}, C_{u}, K_{u}$

P1: Calcule $A_{1}, B_{1}, A_{2}, B_{2}, F$ usando (22)-(24) para $M=M_{a}, C=C_{a}$ e $K=K_{a}$.

P2: Calcule $R_{i j}, i=1, \ldots, i ; j=1, \ldots, p$, usando (25) e (29).

P3: Monte o sistema linear (28) e resolva o problema de mínimos quadrados associado.

P4: Calcule $\Phi$ usando (27) e avalie (17)-(19) para obter $\triangle M, \triangle C, \triangle K$.

P5: Calcule $M_{u}=M_{a}+\triangle M, C_{u}=C_{a}+\triangle C, K_{u}=K_{a}+\triangle K$.

\section{Retorne}

\section{Exemplo numérico}

Considere o sistema $\left(M_{a}, C_{a}, K_{a}\right)$, onde $n=3, p=3, P=3(4) / 2=6$, e

$$
\begin{aligned}
& M_{a}=\left[\begin{array}{lll}
6 & 1 & 0 \\
1 & 5 & 1 \\
0 & 1 & 3
\end{array}\right], C_{a}=\left[\begin{array}{ccc}
10 & 0 & 0 \\
0 & 7 & 0 \\
0 & 0 & 5
\end{array}\right], K_{a}=\left[\begin{array}{ccc}
2.05 & -0.97 & 0 \\
-0.97 & 2.01 & -1.02 \\
& -1.02 & 1 .
\end{array}\right] \\
& X_{1}=\left[\begin{array}{ccc}
-0.5026 & -0.9864 & 0.0116 \\
0.6222 & 0.0159 & 1.0000 \\
-1.0000 & 1.0000 & -0.0273
\end{array}\right], \Lambda_{1}=\left[\begin{array}{ccc}
-1.6979 & 0 & 0 \\
0 & -1.4297 & 0 \\
0 & 0 & -0.9963
\end{array}\right] \\
& Y_{1}=\left[\begin{array}{ccc}
-0.2131 & -0.9891 & 0.2781 \\
0.2447 & 0.8524 & -0.5090 \\
-0.1327 & 0.3147 & -0.0191
\end{array}\right], \Sigma_{1}=\left[\begin{array}{ccc}
-1.80 & 0 & 0 \\
0 & -1.60 & 0 \\
0 & 0 & -1.10
\end{array}\right]
\end{aligned}
$$


e ainda, para fins de comparação,

$$
\Omega\left(\left(M_{a}, C_{a}, K_{a}\right)\right)=\left\{\begin{array}{llllll}
-1.6979 & -1.4297 & -0.9963 & -0.6798 & -0.2372 & -0.0331
\end{array}\right\} .
$$

Algoritmo 3.1 constrói a matriz de coeficientes e o vetor de dados do sistema linear (28):

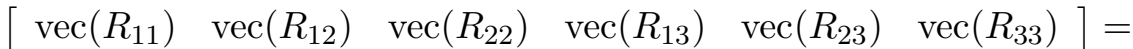

$$
\begin{aligned}
& {\left[\begin{array}{cccccc}
-14.1217 & -31.0308 & -25.9021 & 2.1283 & -2.7410 & 2.0723 \\
5.3640 & 3.4813 & 0.5340 & 13.2542 & 9.7639 & 5.0012 \\
-14.0020 & 4.0710 & 13.3476 & 1.8538 & 7.2190 & 1.9492 \\
-22.7323 & -84.0168 & -153.7445 & -2.3074 & 0.9145 & 8.5190 \\
7.5256 & 16.0881 & 3.2784 & 23.2066 & 56.6797 & 19.0204 \\
-22.5321 & -26.2185 & 79.3250 & -2.6724 & 37.2512 & 8.0691 \\
2.4399 & 8.7738 & 15.7745 & 1.1155 & 2.8809 & -2.5395 \\
-0.2116 & -0.4869 & -0.3871 & -2.2987 & -5.4403 & -4.1191 \\
2.4144 & 2.0334 & -8.1849 & 1.1297 & -6.3484 & -2.4619
\end{array}\right]} \\
& \operatorname{vec}(F)^{T}= \\
& {\left[\begin{array}{lllllllll}
-0.1882 & 0.5944 & 0.3149 & -0.0393 & 1.9893 & 1.5268 & -0.5921 & -0.1201 & -0.0801
\end{array}\right]}
\end{aligned}
$$

e encontramos a solução do problema de mínimos quadrados associado ao sistema de equações algébricas (28) via fatoração QR incompleta com pivotamento de colunas:

$$
\alpha^{T}=\left[\begin{array}{llllll}
.003376 & -.001087 & .006934 & -.009000 & .000248 & .113596
\end{array}\right]
$$

e assim definimos

$$
\Phi=\left[\begin{array}{ccc}
0.003376187 & -0.001086892 & -0.008999070 \\
-0.001086892 & 0.006933552 & 0.000247875 \\
-0.008999070 & 0.000247875 & 0.113596440
\end{array}\right]
$$

e assim, depois de avaliar (17)-(19), obtemos as novas representações matriciais

$$
\begin{gathered}
M_{u}=\left[\begin{array}{lll}
5.3226 & 0.2736 & 0.0328 \\
0.2736 & 2.4180 & 0.3661 \\
0.0328 & 0.3661 & 2.6147
\end{array}\right], C_{u}=\left[\begin{array}{ccc}
10.0287 & 0.1729 & 0.3534 \\
0.1729 & 5.0094 & 0.2956 \\
0.3534 & 0.2956 & 5.1412
\end{array}\right] \\
K_{u}=\left[\begin{array}{ccc}
1.9446 & -0.7957 & -0.0768 \\
-0.7957 & 1.6182 & -0.8168 \\
-0.0768 & -0.8168 & 0.8875
\end{array}\right]
\end{gathered}
$$

que verificam $\left\|M_{u} X_{2} \Lambda_{2}^{2}+C_{u} X_{2} \Lambda_{2}+K_{u} X_{2}\right\|_{F}=3.46320 \cdot 10^{-15}$, mas (o novo espectro foi calculado somente para fins desta comparação)

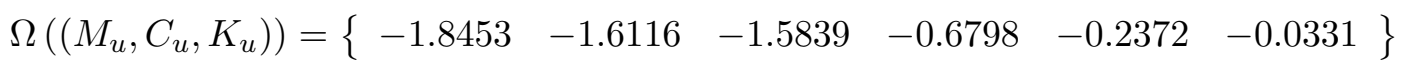

o que indica que foi possível incorporar os dados medidos com alguma (baixa) exatidão.

\section{Conclusão}

No contexto do problema inverso de autovalores e autovetores de sistemas governados por equações diferenciais lineares de segunda ordem, fórmulas para ajuste direto da representação de um sistema linear de vibrações descrito por $\left(M_{a}, C_{a}, K_{a}\right)$, visando incorporar um conjunto medido de frequências naturais e respectivos modos de vibração, sem alterar as demais componentes do espectro, são apresentadas. Diferentemente de muitas estratégias encontradas na literatura, a estratégia apresentada prima por não permitir que o espectro não-medido seja alterado; ao passo que secundariamente, e da melhor maneira possível, segundo um critério de mínimos quadrados, tenta incorporar os dados medidos. O método apresentado é rico em operações com blocos de matrizes, o que permite obter alta performace usando bibliotecas especializadas como LAPACK e BLAS. Exemplo numérico mostra que o método não permite spill-over sobre o espectro não-medido, ao passo que consegue incorporar os dados medidos com alguma exatidão. 


\section{Referências}

[1] J.E. Mottershead, M.I. Friswell, Model updating in structural dynamics: A survey,Journal of Sound and Vibration, 167 (1993) 347-375.

[2] J.E. Mottershead, M.I. Friswell, Finite Element Model updating in Structural Dynamics, Kluwer Academica Publishers, Dordrecht, 1995.

[3] M. Baruch, Optimal correction of mass and stiffness using measured modes, AIAA Journal, 20, (1982), 1623-1626.

[4] A. Berman, E.J. Nagy, Improvement of a large analytical model using testa data, AIAA Journal, 21, (1983), 1168-1173.

[5] B. Caesar, Updating system matrices using modal testing date, in: Proceedings of the fifth IMAC, (1987), pp. 453-459.

[6] M. Link, M. Weiland, J.M. Barragan, Direct physical matrix identification as compared to phase resonance testing: Assessment based on practical application, in: Proceedings of the fifth IMAC, (1987), pp. 804- 811.

[7] D. Zimmerman, M. Widengren, Correcting finite element models using a symmetric eigenstructure assignment technique, AIAA Journal. 28 (1990) 1670-1676.

[8] D. J. Inman, C. Minas, Matching analytical models with experimental modal data in mechanical systems, Control and Dynamics of Systems. 37 (1990) 327-363.

[9] A. M. Kabe, Stiffness matrix adjustment using mode data, AIAA Journal. 23 (1985) 14311436.

[10] B. Caesar, J. Peter, Direct update of dynamic mathematical models from modal test data, AIAA Journal. 25 (1987) 1494-1499.

[11] B. Datta, S. Elhay, and Y.M. Ram, Orthogonality and partial pole assignment for the symmetric definite quadratic pencil, Linear Algebra and Applications, 257 (1997), pp.29-48.

[12] J. Carvalho, B. N. Datta, A. Gupta, M. Lagadapati, A direct method for model updating with incomplete measured data and without spurious modes, Mech. Syst. Signal Process. 21 (2007) 2715-2731.

[13] M. T. Chu, W.-W. Lin, S.-F. Xu, Updating quadratic models with no spill-over effect on unmeasured spectral data, Inverse Problems. 23 (2007) 243-256.

[14] M. T. Chu, B. N. Datta, W.-W. Lin, S.-F. Xu, The spill-over phenomenon in quadratic model updating, AIAA Journal. 46 (2008) 420-428.

[15] D. Chu, M. T. Chu, W.-W. Lin, Quadratic model updating with symmetry, positive definiteness, and no spill-over, SIAM J. Matrix Anal. Appl. 31 (2009) 546-564.

[16] X. Mao, H. Dai, Finite element model updating with positive definiteness and no spill-over, Mech. Syst. Signal Process. 28 (2012) 387-398.

[17] E. Anderson, Z. Bai, C. Bischof, S. Blackford, J. Demmel, J. Dongarra, J. DuCroz, A. Greenbaum, S. Hammerling, A. Mckenney and D. Sorensen. LAPACK User's Guide, Third Edition, SIAM, Philadelphia, 1999.

[18] J. Dongarra, I. Duff, D. Sorensen and H. Van der Vorst. Numerical Linear Algebra for High-Performance Computers. SIAM Press, Philadelphia, 1998. 